\title{
Unravelling the Mechanisms of Interference between Overlapping Resonances
}

\author{
A. García-Vela \\ Instituto de Física Fundamental, Consejo Superior de Investigaciones Científicas, \\ Serrano 123, 28006 Madrid, Spain
}

\begin{abstract}
The enhancement of the resonance lifetime that occurs upon interference of two overlapping resonances excited coherently by two pulses delayed in time, has been investigated as a function of the pulse temporal width and the delay time between the pulses. A general law predicting quantitatively the optimal delay time that maximizes the lifetime enhancement of the two resonances, has been established in terms of the pulse width and of the lifetimes of both resonances when they are excited isolatedly. The specific form of the law and all the results found can be closely related to the characteristic features of the mechanism of interference between the overlapping resonances, providing a detailed understanding on how the mechanism operates. The proposed law is envisioned as a useful tool to design experimental strategies to control the resonance lifetime.

E-mail: garciavela@iff.csic.es
\end{abstract}


Resonance states are very interesting objects of quantum nature that can behave as doorway states from which a variety of molecular processes can be activated. ${ }^{1,2}$ Photodissociation processes like electronic, ${ }^{3}$ vibrational, ${ }^{4,5}$ and rotational ${ }^{6}$ predissociation of a molecular system, as well as low-temperature reactive ${ }^{7-12}$ and nonreactive $\mathrm{e}^{13-20}$ molecular collisions, are examples of processes mediated by resonance states. Control of such molecular processes has been pursued for a long time. ${ }^{21-34}$ In the case of resonance-mediated processes, controlling the behavior of the resonance state (or states) governing the process appears as a real possibility of achieving control of the process of interest. And the behavior of a resonance is determined by the behavior of its properties such as the lifetime and the product fragment state distributions produced upon resonance decay. In this sense, strategies to control both the resonance lifetime ${ }^{35-38}$ and the product fragment distributions ${ }^{39-42}$ have been suggested for overlapping and isolated resonance states.

Many of the schemes proposed to control the resonance properties relay on the manipulation of quantum interference that takes place between overlapping resonances, which are nonorthogonal states, when they are populated simultaneously. This is the case of schemes suggested to control the lifetime of a given resonance state that overlaps with other resonances, by using one, ${ }^{35}$ two, ${ }^{36,37}$ and three ${ }^{37}$ laser pulses to vary the relative population of the different overlapping resonances initially excited. When two pulses with a delay time between them were used to excite two overlapping resonances (each pulse exciting one resonance), the shape of the survival probability and the associated lifetime of one of the resonances (the target resonance) was found to vary when the delay between the pulses was varied, reaching a maximum lifetime at a specific delay time. ${ }^{36} \mathrm{~A}$ similar behavior is expected for the other resonance overlapping with the target one.

Changing the delay time between the pulses causes population of the two resonances at different times, which implies changes in their mechanism of interference and explains variations in the resonance survival probability and lifetime. Now the interesting question is what determines the specific delay time at wich the maximum 
enhancement of the resonance lifetime takes place. Physical intuition indicates that this should be determined by the specific mechanism of interference between the overlapping resonances involved, which should depend on factors like the temporal width of the pulses applied, and the lifetime of the different resonances when they are excited isolatedly (i.e., in the absence of population of the other overlapping resonances). It would be most desirable to have available the knowledge allowing a quantitative prediction of the optimal delay time between pulses leading to maximize the resonance lifetime enhancement for the general case of two overlapping resonances with different (isolated) lifetimes, and for a given temporal width of the exciting pulses. The motivation to reach such a knowledge would be twofold. On the one hand, it would imply a detailed understanding of the mechanisms of quantum interference between overlapping resonances. On the other hand it would provide a valuable and useful tool to simplify the experimental design of a control scheme.

In this work, the quantitative relationship between the optimal delay time between pulses causing maximum lifetime enhancement, and specific conditions of the control scheme designed, like the different lifetimes of the overlapping resonances and the temporal width of the pulses used, is explored in the case of two overlapping resonances. To this purpose, different scenarios have been investigated, varying both the temporal width of the laser pulses applied and the width (and therefore the lifetime) of the two overlapping resonances excited. In this sense, the vibrational predissociation of the $\mathrm{Ne}-\mathrm{Br}_{2}\left(B, v^{\prime}=35\right)$ complex is very convenient to be used as the subject process of this type of study, since this system supports a variety of overlapping resonances with different widths.

Upon laser excitation, $\mathrm{Ne}-\mathrm{Br}_{2}\left(X, v^{\prime \prime}=0\right)+h \nu \rightarrow \mathrm{Ne}^{-\mathrm{Br}_{2}}\left(B, v^{\prime}, n^{\prime}\right)$, an intermolecular van der Waals (vdW) resonance $n^{\prime}$ or a superposition of resonances is populated. The labels $v^{\prime \prime}$ and $v^{\prime}$ denote the vibrational states of $\mathrm{Br}_{2}$ in the $X$ and $B$ electronic states, respectively, while the $n^{\prime}$ index labels the energy position of the resonance, with $n^{\prime}=0$ corresponding to the ground one. Then the resonance excited decays to the fragmentation continuum through vibrational predissociation, 
$\mathrm{Ne}-\mathrm{Br}_{2}\left(B, v^{\prime}, n^{\prime}\right) \rightarrow \mathrm{Ne}+\mathrm{Br}_{2}\left(B, v_{f}<v^{\prime}\right)$. This process has been studied in detail both experimentally ${ }^{43,44}$ and theoretically. ${ }^{4,45}$ The $\mathrm{Ne}_{-} \mathrm{Br}_{2}\left(B, v^{\prime}=35\right)$ excitation with a laser pulse and the subsequent predissociation was simulated with a full threedimensional wave packet method (assuming $J=0$ ) described in detail elsewhere. ${ }^{4,35}$ It is noted that the lifetime calculated with the present model for the decay of the $\mathrm{Ne}-\mathrm{Br}_{2}\left(B, v^{\prime}=16\right)$ ground intermolecular resonance was $69 \mathrm{ps},{ }^{38}$ while the corresponding lifetime estimated experimentally is $68 \pm 3 \mathrm{ps},{ }^{44}$ which assesses the good quality of the model applied to describe this resonance decay process.

The excitation spectrum associated with the ground vdW resonance $n^{\prime}=0$ of $\mathrm{Ne}_{-\mathrm{Br}_{2}}\left(B, v^{\prime}=35, n^{\prime}\right)$ was previously investigated, ${ }^{45}$ and is displayed in Fig. 1 . The

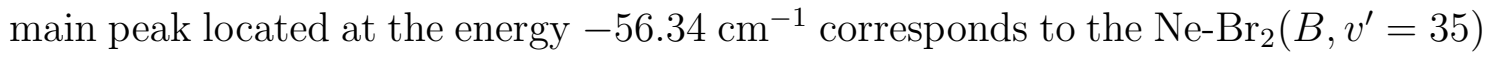
ground resonance, labeled as $\left(v^{\prime}, g r\right)$ in the figure. The spectrum shows several other peaks and bumps at lower and higher energies than that of the main peak, which were identified $^{45}$ as vdW resonances of the $v^{\prime}-1$ lower manifold in the range $n=5-13$ that overlap between them and also with the $v^{\prime}$ ground resonance.

In the simulations carried out, two overlapping resonances are populated simultaneously by a laser field consisting of the combination of two Gaussian pulses, each pulse exciting one of the two resonance states. The two resonances excited are the $n^{\prime}=0$ resonance of the $v^{\prime}=35$ manifold and one vdW resonance of the $v^{\prime}-1$ manifold overlapping with the $v^{\prime}$ ground resonance. In order to analyze the effect of varying the lifetime of the $v^{\prime}-1$ resonance excited, two different resonances have been used in the simulations, namely $n=7$ and 9 , located at -60.76 and -57.75 $\mathrm{cm}^{-1}$, respectively (see Fig. 1). The laser field applied can be expressed as

$$
\mathcal{E}_{\text {pump }}(t)=A_{1} e^{-\left(t-t_{1}\right)^{2} / 2 \sigma^{2}} \cos \left[\omega_{1}\left(t-t_{1}\right)+\phi_{1}\right]+A_{2} e^{-\left(t-t_{2}\right)^{2} / 2 \sigma^{2}} \cos \left[\omega_{2}\left(t-t_{2}\right)+\phi_{2}\right],
$$

where $\omega_{1}$ and $\omega_{2}$ are the photon frequencies required to excite the resonance energy of the $v^{\prime}$ and $v^{\prime}-1$ resonances, respectively, and $t_{1}$ and $t_{2}$ are the time centers of the two pulses. In practice $t_{1}$ is always fixed at the value $t_{1}=0$, and $t_{2}$ is varied. Thus the delay time between the pulses becomes $\Delta t=t_{2}-t_{1}=t_{2}$. For simplicity, in all the simulations it was assumed the same width (related to $\sigma$ ) for the two Gaussian 
pump pulses, and also that $\phi_{1}=\phi_{2}=0$. The width of the pulses was varied in the different simulations. In most of the simulations the amplitudes of the pulses are also assumed to be equal, $A_{1}=A_{2}=1.0 \times 10^{-6}$ a.u., which corresponds to a maximum pulse intensity of about $3.5 \times 10^{4} \mathrm{~W} / \mathrm{cm}^{2}$, within the weak-field regime.

Application of the electric field $\mathcal{E}_{\text {pump }}(t)$ of eqn (1) creates a coherent superposition of the $v^{\prime}\left(\psi_{1}\right)$ and $v^{\prime}-1\left(\psi_{2}\right)$ resonances

$$
\Phi(t)=a_{1}(t) \psi_{1}(t)+a_{2}(t) \psi_{2}(t)
$$

We can express the survival probability associated with the $\psi_{1}$ resonance as

$$
\begin{gathered}
I_{1}(t)=\left|<\psi_{1}\right| \Phi(t)>\left.\right|^{2}=\left|a_{1}(t)<\psi_{1}\right| \psi_{1}(t)>+a_{2}(t)<\psi_{1}\left|\psi_{2}(t)>\right|^{2}= \\
\left|a_{1}(t)\right|^{2}\left|<\psi_{1}\right| \psi_{1}(t)>\left.\right|^{2}+\left|a_{2}(t)\right|^{2}\left|<\psi_{1}\right| \psi_{2}(t)>\left.\right|^{2}+ \\
a_{1}(t) a_{2}(t)^{*}<\psi_{1}\left|\psi_{1}(t)><\psi_{2}(t)\right| \psi_{1}>+a_{1}(t)^{*} a_{2}(t)<\psi_{1}(t)\left|\psi_{1}><\psi_{1}\right| \psi_{2}(t)>,
\end{gathered}
$$

and similarly for the $\psi_{2}$ resonance

$$
\begin{gathered}
I_{2}(t)=\left|a_{2}(t)\right|^{2}\left|<\psi_{2}\right| \psi_{2}(t)>\left.\right|^{2}+\left|a_{1}(t)\right|^{2}\left|<\psi_{2}\right| \psi_{1}(t)>\left.\right|^{2}+ \\
a_{1}(t) a_{2}^{*}(t)<\psi_{2}\left|\psi_{1}(t)><\psi_{2}(t)\right| \psi_{2}>+a_{1}^{*}(t) a_{2}(t)<\psi_{1}(t)\left|\psi_{2}><\psi_{2}\right| \psi_{2}(t)>.
\end{gathered}
$$

The first term of the right hand sides (rhs) of eqns (3) $\left(\left|a_{1}(t)\right|^{2}\left|<\psi_{1}\right| \psi_{1}(t)>\left.\right|^{2}\right)$ and (4) $\left(\left|a_{2}(t)\right|^{2}\left|<\psi_{2}\right| \psi_{2}(t)>\left.\right|^{2}\right)$ is the square of the autocorrelation function of $\psi_{1}$ and $\psi_{2}$, respectively. This is the traditional definition of the survival probability for an isolated, nonoverlapping resonance. Actually, survival probabilities of the form $I_{1}(t)=\left|a_{1}(t)\right|^{2}\left|<\psi_{1}\right| \psi_{1}(t)>\left.\right|^{2}$ or $I_{2}(t)=\left|a_{2}(t)\right|^{2}\left|<\psi_{2}\right| \psi_{2}(t)>\left.\right|^{2}$ is what one would obtain if, instead of exciting simultaneously both $\psi_{1}$ and $\psi_{2}$ in the superposition of eqn (2) (i.e., by making $a_{1}(t)$ and $a_{2}(t)$ simultaneously nonzero), one excites only $\psi_{1}\left(\right.$ with $\left.a_{2}(t)=0\right)$ or $\psi_{2}\left(\right.$ with $\left.a_{1}(t)=0\right)$, by using a single pulse with a relatively narrow spectral width around the resonance energy of either $\psi_{1}$ or $\psi_{2}$. In this case the shape of $I_{1}(t)$ and $I_{2}(t)$ would be the convolution of the single Gaussian pulse envelope (the $\left|a_{n}(t)\right|^{2}$ dependence of $\left.I_{n}(t), n=1,2\right)$ with the resonance exponential 
decay (the $\left|<\psi_{n}\right| \psi_{n}(t)>\left.\right|^{2}$ dependence). The lifetime associated with $I_{n}(t)$ in this case would be the "isolated resonance lifetime", $\tau_{n}^{i s o}$.

However, when $\psi_{1}$ and $\psi_{2}$ are populated simultaneously $\left(a_{1}(t) \neq 0\right.$ and $a_{2}(t) \neq$ 0 ), since they overlap (i.e., $<\psi_{1} \mid \psi_{2}(t)>\neq 0$ and $<\psi_{2} \mid \psi_{1}(t)>\neq 0$ ), three additional terms originated by quantum interference appear in eqns (3) and (4), and contribute to the survival probabilities $I_{1}(t)$ and $I_{2}(t)$. The effect of these interference terms is "dressing" the isolated resonance survival probabilities $I_{n}(t)=\left|a_{n}(t)\right|^{2} \mid<$ $\psi_{n}\left|\psi_{n}(t)>\right|^{2}$, and therefore changing their shape. As a result of this change in shape, the lifetime associated with $I_{n}(t)$ also changes from $\tau_{n}^{i s o}$ to a different value. By varying the delay $\Delta t$ between the pulses, the mechanism of interference between the resonances is modified, which causes variation of the interference terms and therefore a change in the $I_{n}(t)$ shape and in the associated lifetime.

The following strategy was adopted in the simulations. Once a specific set of two overlapping resonances is chosen (either $\left(\psi_{1}, \psi_{2}\right)=\left(n^{\prime}=0, n=9\right)$ or $\left(\psi_{1}, \psi_{2}\right)=$ $\left(n^{\prime}=0, n=7\right)$ ), a superposition of them is prepared with the field of eqn (1) with a given $\Delta t$. The time evolution of this wave packet is computed and the $I_{1}(t)$ and $I_{2}(t)$ curves are calculated. In order to extract the lifetimes associated with both curves, they are fitted to the function ${ }^{4,43}$

$$
I_{n}\left(t_{j}\right)=A \int_{-\infty}^{t_{j}} C C(t)\left[\exp \left(-\left(t_{j}-t\right) / \tau_{n}\right)\right] d t
$$

being $C C(t)$ the laser cross-correlation curve and $A$ an amplitude scaling parameter. In this way two lifetimes, $\tau_{1}$ and $\tau_{2}$, are obtained for the two resonances in the specific conditions of $\Delta t$ and the temporal width of the pulses used. Fig. 2 shows an example of a typical fit, of $I_{1}(t)$ in this case to obtain $\tau_{1}$, using eqn (5). The $I_{1}(t)$ curve displays pronounced oscillations caused by the interference terms of eqn (3). Simulations are carried out for different $\Delta t$ values, providing $\tau_{1}(\Delta t)$ and $\tau_{2}(\Delta t)$ functions that display a maximum. The above procedure is repeated by changing the pulse width and the set of two overlapping resonances.

In the simulations three different temporal widths have been used for the pulses of eqn (1), namely FWHM=100, 200, and 300 ps, with associated spectral widths 
of $0.3,0.15$, and $0.1 \mathrm{~cm}^{-1}$, respectively. These narrow bandwidths ensure that each pulse of eqn (1) excites essentially a single resonance. Simulations were carried out for the two sets $\left(n^{\prime}=0, n=9\right)$ and $\left(n^{\prime}=0, n=7\right)$ of resonances with the above pulses, and the resulting $\tau_{1}(\Delta t)$ and $\tau_{2}(\Delta t)$ functions are shown in Fig. 3.

Additional simulations with a single pulse field (with $A_{1}=0$ or $A_{2}=0$ in eqn (1)) were performed for the two sets of resonances and the pulse widths used. They provide the $\tau_{n}^{i s o}$ lifetimes associated with the three resonances used $\left(n^{\prime}=0\right.$, $n=7$, and 9). The lifetimes obtained are $\tau_{1}^{i s o}=3.8 \mathrm{ps}$ for the $\left(v^{\prime}=35, n^{\prime}=0\right)$ ground resonance, and $\tau_{2}^{i s o}=1.0$ and $13.0 \mathrm{ps}$ for the $v^{\prime}-1 n=7$ and 9 resonances, respectively. This is done in order to check that the $\tau_{n}^{i s o}$ values coincide with the corresponding $\tau_{n}$ values when $|\Delta t|$ is very large and the two resonances are excited in an isolated way, which is confirmed from the results of Fig. 3.

The first interesting result of Fig. 3 is that in all cases studied the maximum value of both $\tau_{1}(\Delta t)$ and $\tau_{2}(\Delta t)$ occurs at the same delay $\Delta t$. This result, not expected a priori, appears to be related to the nature of the mechanism of interference between overlapping resonances. Specifically, the maximum lifetime values obtained for the $\left(n^{\prime}=0, n=9\right)$ set of resonances are $\tau_{1}^{\max }=21.0,61.0$, and $65.0 \mathrm{ps}$, and $\tau_{2}^{\max }=17.0$, 46.0, and $53.0 \mathrm{ps}$, for the pulse widths of FWHM=100, 200, and $300 \mathrm{ps}$, respectively, and they were found at the delays $\Delta t=65,120$, and $165 \mathrm{ps}$, respectively. For the $\left(n^{\prime}=0, n=7\right)$ resonances the maximum lifetimes are $\tau_{1}^{\max }=37.0 \mathrm{ps}$ and $\tau_{2}^{\max }=3.3$ ps for $\mathrm{FWHM}=200 \mathrm{ps}$, found at the delay $\Delta t=105 \mathrm{ps}$. Another interesting result arises from the comparison of the maximum lifetimes obtained for the three pulse widths for the $\left(n^{\prime}=0, n=9\right)$ resonances. The maximum value of both $\tau_{1}(\Delta t)$ and $\tau_{2}(\Delta t)$ increases with increasing temporal width of the pulses. This increase, however, appears to reach a saturation at some point, since the lifetime enhancement when changing from FWHM=200 to 300 ps is significantly smaller than when going from FWHM=100 to 200 ps. The increase of maximum lifetime enhancement with increasing pulse temporal width again seems to be related to the mechanism of resonance interference. This point will be discussed in more detail below. 
In the results of Fig. 3 the pulse delay at which the maximum lifetime enhancement occurs, $\Delta t_{\max }$, is obtained by varying both the temporal width of the pulses and the lifetime $\tau_{2}^{i s o}$ of the $v^{\prime}-1$ resonances (changing from $n=9$ to $n=7$ ), thus making possible to establish empirically a quantitative correlation between $\Delta t_{\max }$ and these two parameters. It is found that the law $\Delta t_{\max }=F W H M / 2+\tau_{1}^{i s o}+\tau_{2}^{i s o}$ fits nicely all the four values of $\Delta t_{\max }$ of Fig. 3. Thus, in the general case of two overlapping resonances, each of them excited by a Gaussian pulse, both pulses with the same temporal width and a delay time, one can determine the delay at which the maximum lifetime enhancement of the two resonances will occur by simply adding the isolated lifetimes of both resonances plus half the temporal width of the pulses.

An intriguing question now is why in all cases studied $\Delta t_{\max }$ is positive, while for negative $\Delta t$ values the enhancement of the $\tau_{n}$ lifetimes is rather low. With the convention adopted here that $t_{1}=0$ and that $\Delta t=t_{2}-t_{1}=t_{2}$, an equivalent question is whether $\Delta t_{\max }$ is determined by the order in which the two resonances are excited by the field of eqn (1). This point is analyzed in the following.

In Fig. 1 the peak associated with the ground resonance $n^{\prime}=0$ is more intense than the peaks associated with the $v^{\prime}-1 n=7$ and 9 resonances. Thus, in the case of $A_{1}=A_{2}$ in eqn (1), the $n^{\prime}=0$ resonance is populated with more intensity than $n=7$ and 9. Since quantum interference between the two resonances is determined by the amount of wave packet amplitude pumped to each resonance at a given time, the intensity of population of each resonance might determine the order of excitation of the resonances leading to $\Delta t_{\max }$, and therefore its sign. In order to check this point, a simulation was carried out for the $\left(n^{\prime}=0, n=9\right)$ set of resonances, applying the laser field of eqn (1) with a width FWHM=200 ps, but with the pulse amplitudes $A_{2}=3 A_{1}$ instead of $A_{2}=A_{1}$, which now ensures that the $n=9$ resonance is populated with more intensity than $n^{\prime}=0$. The convention $t_{1}=0$ and $\Delta t=t_{2}$ was still kept. The calculated $\tau_{1}(\Delta t)$ and $\tau_{2}(\Delta t)$ functions are displayed in Fig. 4.

Most interestingly, the maximum lifetime values of $\tau_{1}^{\max }=28.0 \mathrm{ps}$ and $\tau_{2}^{\max }=$ $30.0 \mathrm{ps}$ were found for the negative delay time $\Delta t_{\max }=-120 \mathrm{ps}$. The absolute 
value $\left|\Delta t_{\text {max }}\right|=120$ ps coincides with that previously found for FWHM=200 ps and $A_{2}=A_{1}$, i.e., when the $n^{\prime}=0$ resonance was populated with more intensity than $n=9$. The first implication of this result is that the relative intensity of population of the two resonances indeed determines the resonance excitation sequence leading to $\Delta t_{\max }: \Delta t_{\max }$ occurs when the most intensely populated resonance is excited first. Depending on the specific definition of $\Delta t$ (i.e., either $\Delta t=t_{2}-t_{1}$ or $\Delta t=t_{1}-t_{2}$ ), and on the assignment of the pulses centered at $t_{1}$ and $t_{2}$ to each resonance, the sign of $\Delta t_{\max }$ will be positive or negative. Thus, we can reformulate the previous law as

$$
\Delta t_{\max }=a\left(F W H M / 2+\tau_{1}^{i s o}+\tau_{2}^{i s o}\right)
$$

where $a$ stands for the sign of $\Delta t_{\max }, a= \pm 1$. For the definition $\Delta t=t_{2}-t_{1}, a=1$ when the pulse centered at $t_{1}$ excite the most populated resonance, and $a=-1$ when this resonance is excited by the pulse centered at $t_{2}$.

The correct prediction of $\Delta t_{\max }$ by eqn (6) and the coincidence of the result $\left|\Delta t_{\text {max }}\right|=120 \mathrm{ps}$ in the two cases of $A_{2}=A_{1}$ and $A_{2}=3 A_{1}$ for FWHM=200 ps, seems to indicate that the value of $\left|\Delta t_{\max }\right|$ is not determined (at least strongly) by the specific relative population of the two resonances. Another interesting result of Fig. 4 is that $\tau_{2}^{\max }>\tau_{1}^{\max }$, which is the opposite result to that found in Fig. 3, when the $\psi_{1}$ resonance was populated with more intensity. This result would imply that interference between the two resonances would favor a larger enhancement of the lifetime of the resonance populated with the largest intensity.

It is now interesting to rationalize all the previous results in relation with the characteristics of the mechanism of interference between the two overlapping resonances. For this analysis, the survival probabilities of the $n^{\prime}=0$ and $n=9$ resonances will be used when they are excited in the absence of population of the other resonance (i.e., when $A_{2}=0$ and $A_{1}=0$, respectively) with a pulse with FWHM $=200 \mathrm{ps}$, and with $A_{2}=A_{1}$. While what actually interferes is the amplitude pumped to each resonance, the $I_{n}(t)$ curves will similarly serve to illustrate how the interference mechanism operates. Since each resonance is excited isolatedly, no interference is possible, and the $I_{n}(t)$ curves of Fig. 5 do not show oscillations. 
Resonance interference was found to be most effective in order to enhance the resonance lifetime when it occurs in the time region of the exponential decay of $I_{n}(t) \cdot{ }^{36}$ Taking this into account, one can understand from Fig. 5 the result of $\Delta t_{\max }=120$ ps found in Fig. 3, when the most populated resonance is excited first, vs. the other possibility of $\Delta t=-120 \mathrm{ps}$. Indeed, for $\Delta t=120$ ps the $I_{1}(t)$ and $I_{2}(t)$ curves overlap along time in a more favorable way, maximizing the amount of simultaneous population in both resonances in the time region of the decay of the two resonances, then maximizing the intensity of interference in that region. This explains the result that the same $\Delta t_{\max }$ leads to maximum lifetime enhancement for both $\tau_{1}$ and $\tau_{2}$. For $\Delta t=-120$ ps the time overlap between resonance populations is much less favorable for interference, leading to rather poor lifetime enhancements, as shown in Fig. 3. Maximizing the time overlap between the resonance populations in the appropriate time region also explains the dependence of $\Delta t_{\max }$ on the pulse width and on the sum of $\tau_{1}^{i s o}$ and $\tau_{2}^{i s o}$. Indeed, as both the pulse width and the lifetimes $\tau_{n}^{i s o}$ increase, it takes a longer time to reach the region of the resonance decay, what increases $\Delta t_{\max }$ and explains the specific form of eqn (6). In addition, as the pulse width increases the time range of interference in the optimal region becomes longer, leading to a larger enhancement of both $\tau_{n}^{\max }$, as found in Fig. 3 . The simple form of eqn (6) appears to reflect the relatively simple physics underlying the mechanism of interference between overlapping resonances.

In summary, the enhancement of the resonance lifetime that occurs upon interference of two overlapping resonances excited coherently by two pulses delayed in time, has been investigated as a function of the pulse temporal width and the delay time between the pulses. A general law that predicts quantitatively the optimal delay time between pulses that maximizes the lifetime enhancement of the two resonances has been established. This optimal delay time depends on the pulse width and on the lifetimes of the two resonances when they are excited isolatedly. The specific form of the law and the results found are readily explained in terms of the features of the mechanism of interference between the resonances, providing a physical basis for 
the law and a detailed understanding on how the mechanism operates. Finally, the present findings are general to any situation of two overlapping resonances excited by two delayed pulses, and are envisioned as a useful tool to design experimental strategies to control the resonance lifetime.

\section{Conflicts of interest}

There are no conflicts of interest to declare.

\section{Acknowledgements}

This work was funded by the Ministerio de Economía y Competitividad (MINECO, Spain), Grant No. CTQ2015-65033-P, and COST Action program, grant No. CM1401. The Centro de Supercomputación de Galicia (CESGA, Spain) is acknowledged for the use of its resources.

\section{Notes and references}

(1) D. W. Chandler, J. Chem. Phys., 2010, 132, 110901.

(2) N. Balakrishnan, J. Chem. Phys., 2016, 145, 150901.

(3) G. Balerdi, J. Woodhouse, A. Zanchet, R. de Nalda, M. L. Senent, A. GarcíaVela and L. Bañares, Phys. Chem. Chem. Phys., 2016, 18, 110.

(4) A. García-Vela and K. C. Janda, J. Chem. Phys., 2006, 124, 034305.

(5) A. García-Vela, J. Chem. Phys., 2008, 129, 094307.

(6) C. M. Lovejoy and D. J. Nesbitt, J. Chem. Phys., 1990, 93, 5387.

(7) R. T. Skodje, D. Skouteris, D. E. Manolopoulos, S. H. Lee, F. Dong and K. Liu, Phys. Rev. Lett., 2000, 85, 1206.

(8) M. Qiu et al., Science, 2006, 311, 1440.

(9) L. Che et al., Science, 2007, 317, 1061.

(10) J. B. Kim et al., Science, 2015, 349, 510.

(11) W. Shiu, J. J. Lin and K. Liu, Phys. Rev. Lett., 2004, 92, 103201.

(12) T. Westermann et al., Angew. Chem. Int. Ed., 2014, 53, 1122. 
(13) S. Chefdeville et al., Phys. Rev. Lett., 2012, 109, 023201.

(14) A. B. Henson, S. Gersten, Y. Shagam, J. Narevicius and E. Narevicius, Science, 2012, 338, 234.

(15) E. Lavert-Ofir et al., Nat. Chem., 2014, 6, 332.

(16) S. N. Vogels et al., Phys. Rev. Lett., 2014, 113, 263202.

(17) S. N. Vogels et al., Science, 2015, 350, 787.

(18) J. Jankunas, K. Jachymski, M. Hapka and A. Osterwalder, J. Chem. Phys., 2015, 142, 164305 .

(19) A. Bergeat, J. Onvlee, C. Naulin, A. van der Avoird and M. Costes, Nat. Chem., 2015, 7, 349.

(20) C. Naulin and M. Costes, Chem. Sci, 2016, 7, 2462.

(21) D. Tannor and S. Rice, J. Chem. Phys., 1985, 83, 5013.

(22) P. Brumer and M. Shapiro, Chem. Phys. Lett., 1986, 126, 541.

(23) A. Assion, T. Baumert, M. Bergt, T. Brixner, B. Kiefer, V. Seyfried, M. Strehle and G. Gerber, Science, 1998, 282, 919.

(24) P. Anfinrud, R. de Vivie-Riedle and V. Engel, Proc. Natl. Acad. Sci. USA, 1999, 96, 8328-8329.

(25) R. J. Levis, G. M. Menkir and H. Rabitz, Science, 2001, 292, 709.

(26) E. Skovsen, M. Machholm, T. Ejdrup, J. Thфgersen and H. Stapelfeldt, Phys. Rev. Lett., 2002, 89, 133004.

(27) C. Daniel, J. Full, L. González, C. Lupulescu, J. Manz, A. Merli, S. Vajda and L. Wöste, Science, 2003, 299, 536.

(28) B. J. Sussman, D. Townsend, M. I. Ivanov and A. Stolow, Science, 2006, $314,278$.

(29) G. Katz, M. A. Ratner and R. Kosloff, Phys. Rev. Lett., 2007, 98, 203006.

(30) M. E. Corrales, J. González-Vázquez, G. Balerdi, I. R. Solá, R. de Nalda and L. Bañares, Nat. Chem., 2014, 6, 785.

(31) L. Belshaw, F. Calegari, M. J. Duffy, A. Trabattoni, L. Poletto, M. Nisoli and J. B. Greenwood, J. Phys. Chem. Lett., 2012, 3, 3751. 
(32) M. Shapiro and P. Brumer, Quantum Control of Molecular Processes, Second Edition, Wiley-VCH verlag GmbH and Co. KGaA, Singapore, 2012.

(33) F. Calegari et al., Science, 2014, 346, 336.

(34) R. Cireasa et al., Nat. Phys., 2015, 11, 654.

(35) A. García-Vela, J. Chem. Phys., 2012, 136, 134304.

(36) A. García-Vela, J. Phys. Chem. Lett., 2012, 3, 1941.

(37) A. García-Vela, J. Chem. Phys., 2013, 139, 134306.

(38) A. García-Vela, Phys. Chem. Chem. Phys., 2015, 17, 29072.

(39) A. García-Vela and N. E. Henriksen, J. Phys. Chem. Lett., 2015, 6, 824.

(40) A. García-Vela, Phys. Chem. Chem. Phys., 2016, 18, 10346.

(41) A. García-Vela, J. Chem. Phys., 2016, 144, 141102.

(42) A. García-Vela, Chem. Sci., 2017, 8, 4804.

(43) J. A. Cabrera, C. R. Bieler, B. C. Olbricht, W. E. van der Veer and K. C. Janda, J. Chem. Phys., 2005, 123, 054311.

(44) M. A. Taylor, J. M. Pio, W. E. van der Veer and K. C. Janda, J. Chem. Phys., 2010, 132, 104309.

(45) A. García-Vela, J. Chem. Phys., 2007, 126, 124306. 


\section{FIGURE CAPTIONS}

Fig. 1. Calculated excitation spectrum associated with the ground vdW resonance of $\mathrm{Ne}-\mathrm{Br}_{2}\left(B, v^{\prime}=35\right)$. The energy axis is relative to the $\mathrm{Ne}+\mathrm{Br}_{2}\left(B, v^{\prime}=35, j^{\prime}=\right.$ 0 ) dissociation threshold. The main peak of the spectrum (labeled as $\left(v^{\prime}, g r\right)$ ) corresponds to the ground resonance of the $v^{\prime}=35$ vibrational manifold. Resonances associated with the $v^{\prime}-1$ manifold in the range $n=5-13$ have been identified, and their positions are indicated by arrows in the figure.

Fig. 2. Survival probability $I_{1}(t)$ of the $n^{\prime}=0$ ground resonace of $v^{\prime}=35$ when the set $\left(\psi_{1}, \psi_{2}\right)=\left(n^{\prime}=0, n=9\right)$ of two overlapping resonances is excited with the laser field of eqn (1) with pulses having a width FWHM=200 ps, and a delay time between pulses $\Delta t=80 \mathrm{ps}$. The plain, nonoscillating curve is a fit to $I_{1}(t)$ obtained using eqn (5).

Fig. 3. Calculated lifetimes of the two overlapping resonances vs. the delay time between the two pulses exciting the set of resonances $\left(n^{\prime}=0, n=9\right)$ by applying the pulse width of FWHM=100 ps (left upper panel), FWHM=200 ps (right upper panel), FWHM=300 ps (left lower panel), and exciting the set of resonances $\left(n^{\prime}=0, n=7\right)$ by applying the pulse width of $\mathrm{FWHM}=200 \mathrm{ps}$ (right lower panel). In all cases the ratio $A_{2}=A_{1}$ of pulse amplitudes was used in eqn (1).

Fig. 4. Calculated lifetimes of the $n^{\prime}=0$ and $n=9$ resonances vs. the delay time between the two exciting pulses, by applying the pulse width of FWHM=200 ps. The ratio $A_{2}=3 A_{1}$ of pulse amplitudes was used in eqn (1).

Fig. 5. Survival probabilities $I_{1}(t)$ and $I_{2}(t)$ of the $n^{\prime}=0$ and $n=9$ resonances, respectively, when they are excited isolatedly using a pulse width of FWHM=200 ps. In the case of $n^{\prime}=0$ the exciting pulse is centered at $t_{1}=0$, while for $n=9$ the pulse was centered at $t_{2}=-120$ and $120 \mathrm{ps}$. 


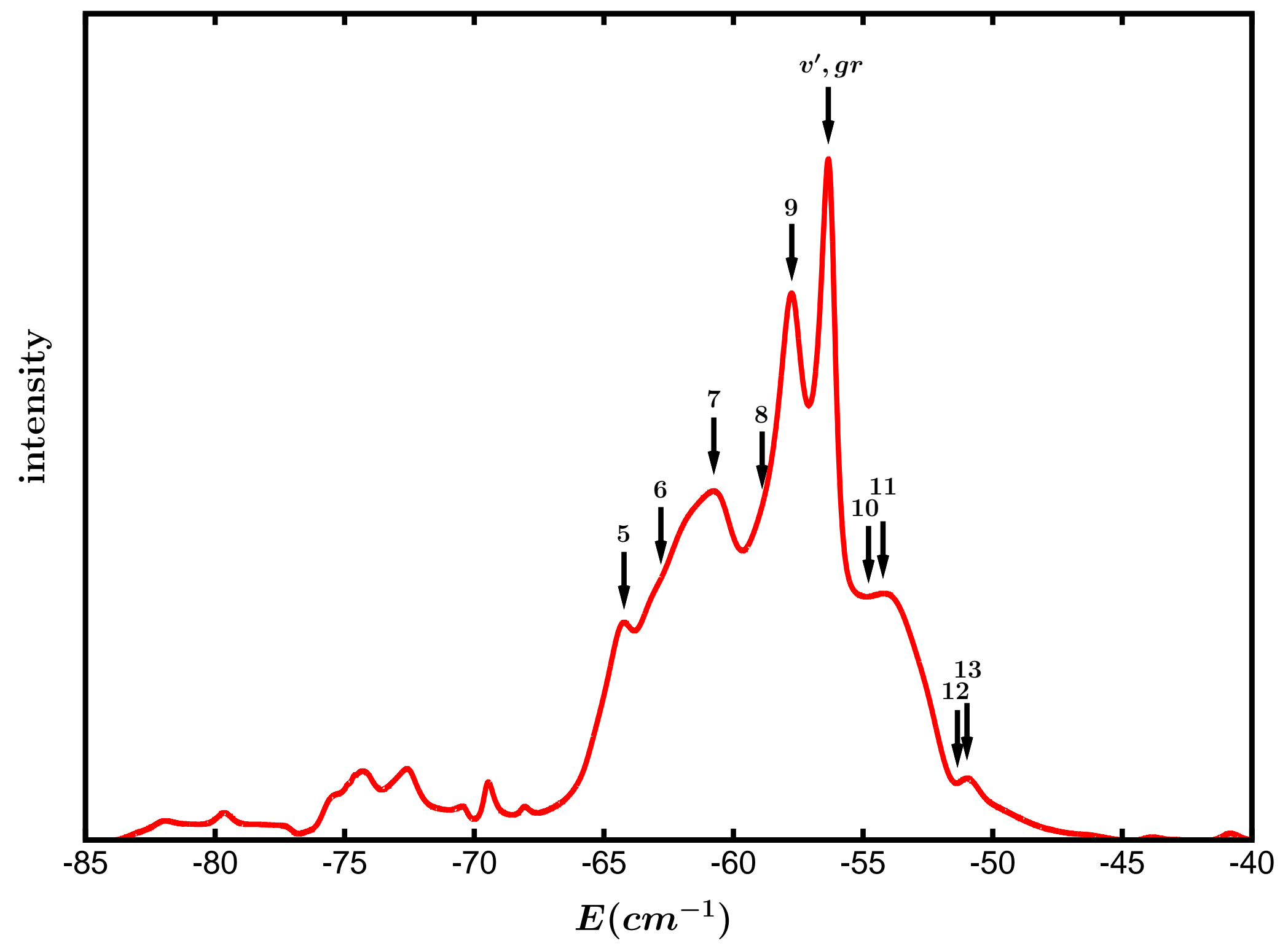

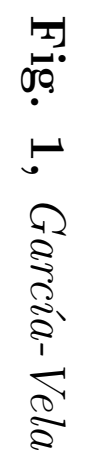


Fig. 2, García-Vela

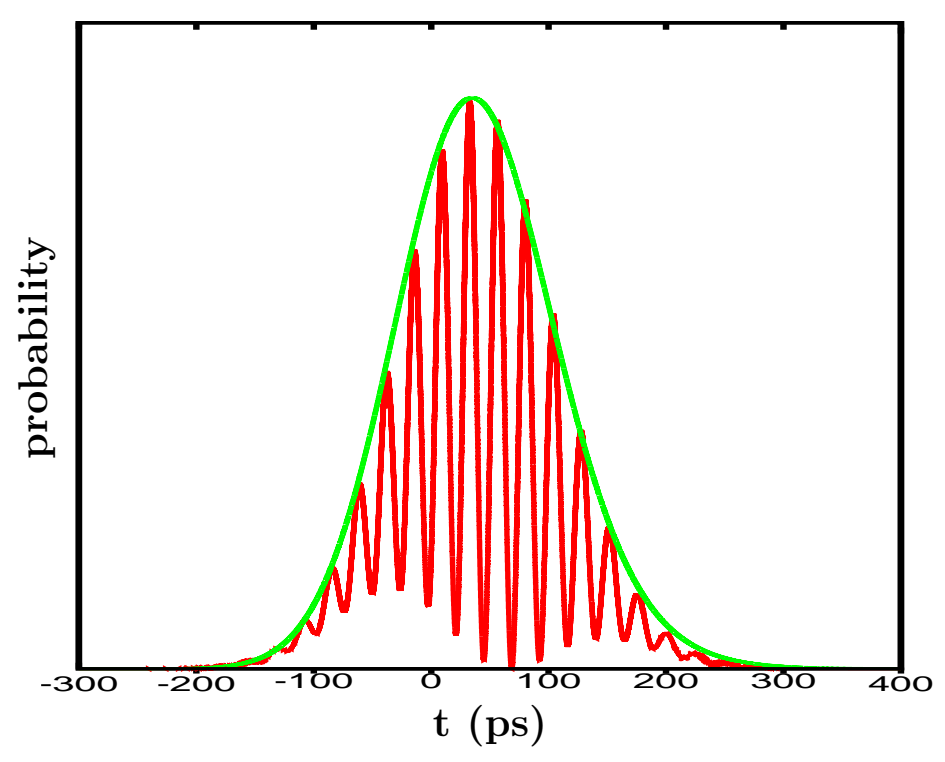


Fig. 3, García-Vela
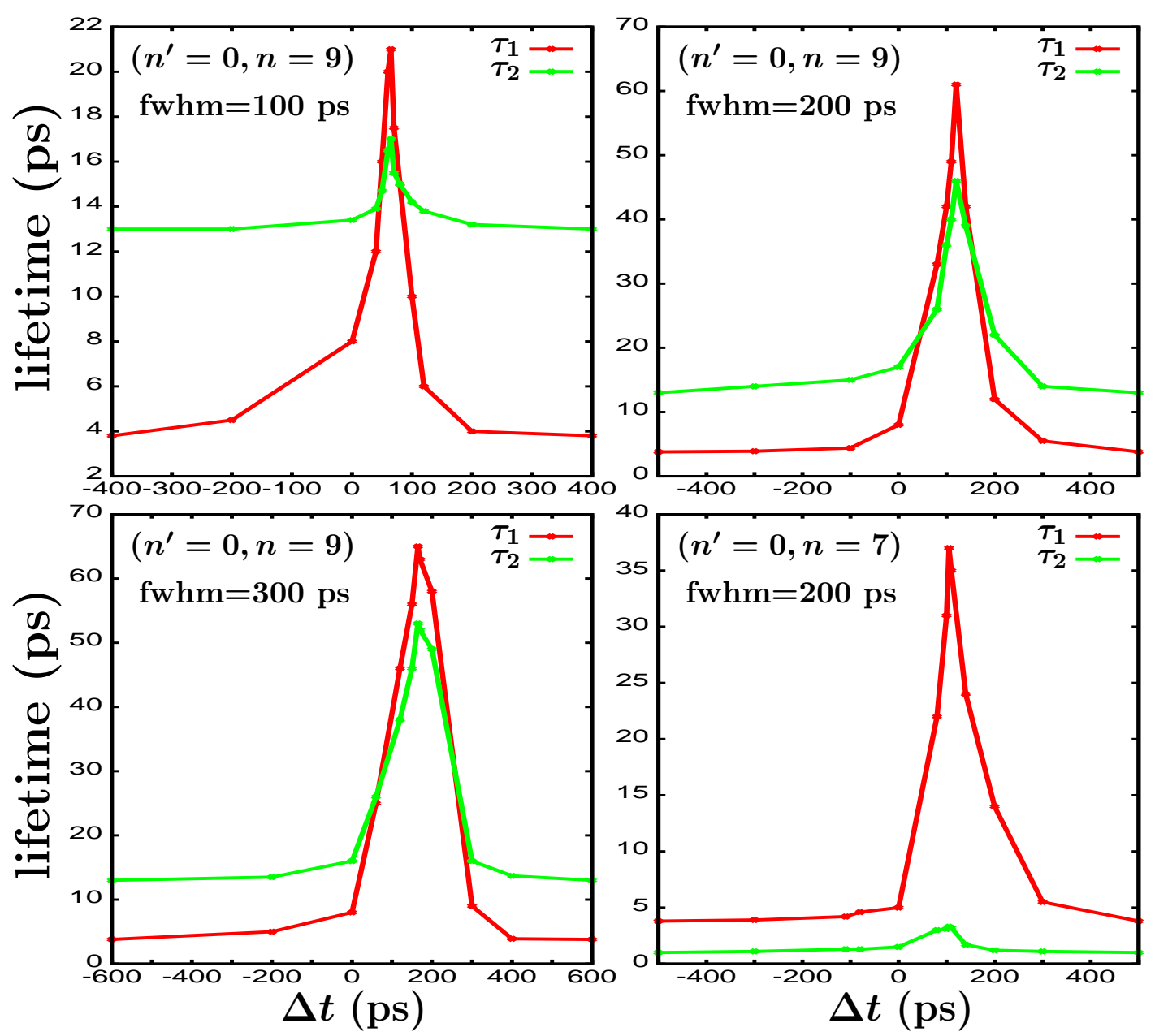
Fig. 4, García-Vela

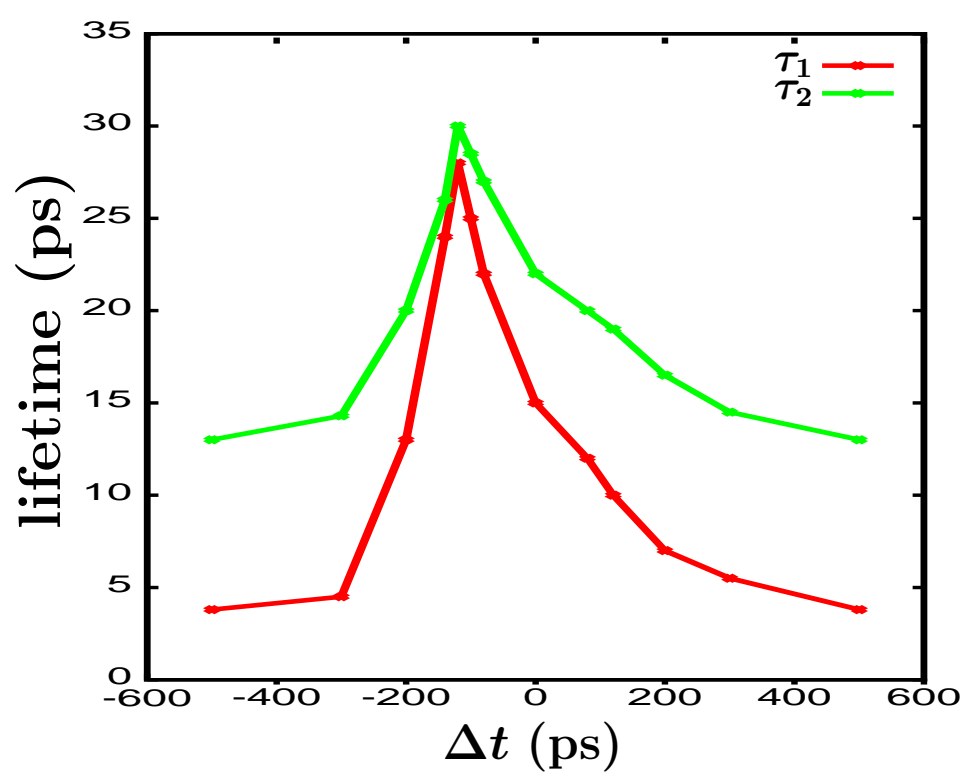


Fig. 5, García-Vela

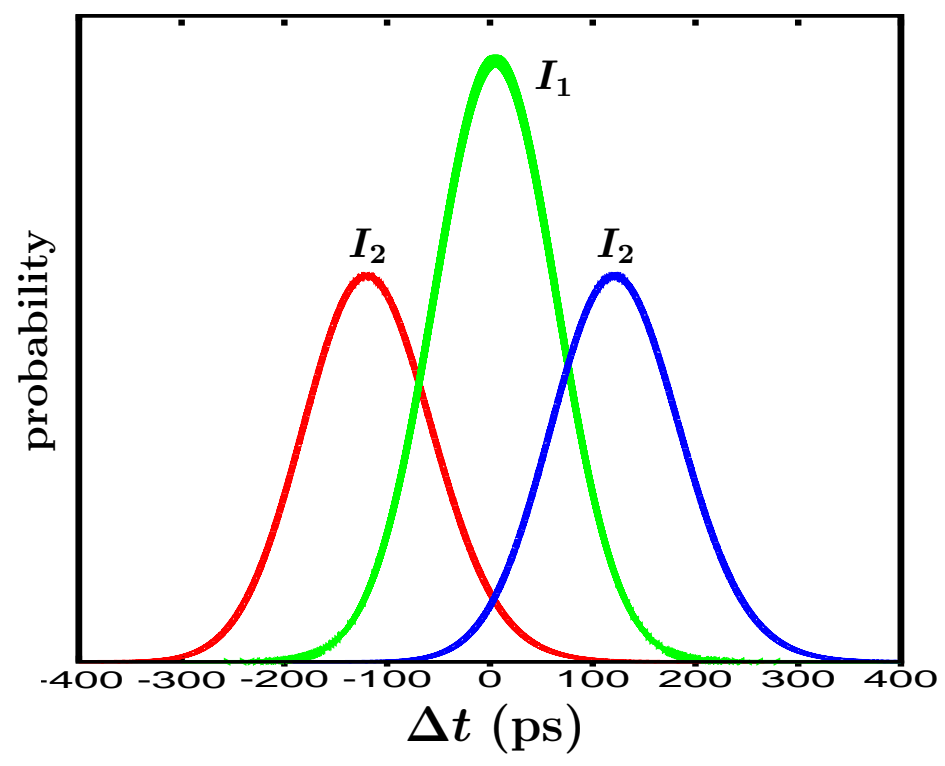

